\title{
Temporal spatial expression and function of non-muscle myosin II isoforms IIA and IIB in scar remodeling
}

\author{
Jennifer E Bond ${ }^{1}$, Trung Q Ho ${ }^{1}$, Maria Angelica Selim² ${ }^{2}$, Cedric L Hunter', Edith V Bowers ${ }^{2}$ and Howard Levinson ${ }^{1,2}$
}

Scar contracture is believed to be caused by the cell contractility during the remodeling phase of wound healing. Cell contractility is mediated by non-muscle myosin II (NMMII) and actin, but the temporal-spatial expression profile of NMMII isoforms A and B (IIA and IIB) during the remodeling phase and the role of NMMII in scar fibroblast tissue remodeling are unknown. Human scar tissue immunostained for IIA and IIB showed that both isoforms were highly expressed in scar tissue throughout the remodeling phase of repair and expression levels returned to normal after the remodeling phase. Human scar tissue immunostained for $\beta-, \gamma$ - and $\alpha$-smooth muscle actin showed that all isoforms were consistently expressed throughout the remodeling phase of repair. The $\beta$ - and $\gamma$-smooth muscle actin were widely expressed throughout the dermis, but $\alpha$-smooth muscle actin was only locally expressed within the dermis. In vitro, fibroblasts explanted from scar tissue were shown to express more IIA than fibroblasts explanted from normal tissue and scar fibroblasts contracted collagen lattices to a greater extent than normal fibroblasts. Blebbistatin was used to demonstrate the function of NMMII in collagen lattice contraction. In normal tissue, fibroblasts are stress-shielded from external tensile stress by the extracellular matrix. After dermal injury and during remodeling, fibroblasts are exposed to a matrix of increased stiffness. The effect of matrix stiffness on IIA and IIB expression was examined. IIA expression was greater in fibroblasts cultured in collagen lattices with increasing stiffness, and in fibroblasts cultured on glass slides compared with polyacrylamide gels with stiffness of $1 \mathrm{kPa}$. In conclusion, NMMII and actin isoform expression changes coordinately with the remodeling phase of repair, and NMMII is increased as matrix stiffness increases. As NMMII expression increases, so does the fibroblast contractility.

Laboratory Investigation (2011) 91, 499-508; doi:10.1038/labinvest.2010.181; published online 22 November 2010

KEYWORDS: non-muscle myosin II; scar contracture; tissue remodeling

Scar contracture is a highly prevalent disorder that affects all organs of the human body regardless of whether the primary mechanism of injury is idiopathic (keloids and Dupuytren's disease), desmoplastic (cancer), autoimmune (scleroderma), inflammatory (Crohn's disease), infectious (cirrhosis) or traumatic (cicatricial ectropion). In terms of the number of patients affected by cutaneous scarring, there are estimated to be more than 2.4 million Americans scarred by burns every year, and more than 40 million scar victims worldwide who are affected by scarring in the skin. ${ }^{1}$ Despite the highly prevalent nature of this problem, current methods to prevent contracture formation are relatively ineffective and the pathogenetic mechanisms of tissue remodeling are not well understood. $^{2}$

Wound healing is classically divided into three sequential, overlapping phases of repair: inflammation, proliferation and remodeling. The inflammatory phase lasts for 3 days, the proliferative phase for 2 weeks and the remodeling phase of repair begins on day 14 after wounding and lasts for 6 months or in rare instances up to two years. ${ }^{3}$ The tensile strength of an incisional cutaneous wound 2 weeks after wounding is approximately $10 \%$ of normal uninjured skin. ${ }^{4}$ It is during the ensuing 6 months of the remodeling phase of repair that wounds regain tensile strength, returning to $80 \%$ of normal skin. It is thought that the increase in tensile strength is a result of four processes: cell contractility, de novo collagen production, metalloproteinase secretion and collagen crosslinking. ${ }^{5-7}$ Of the four processes, cell contractility is considered to be the singular action that is primarily causative of scar contracture formation as fibroblasts and myofibroblasts exert incremental, progressive tractional forces in slip-ratchet-like fashion. ${ }^{8}$ It is unclear however, what

\footnotetext{
${ }^{1}$ Division of Plastic and Reconstructive Surgery, Department of Surgery, Duke University Medical Center, Durham, NC, USA and ${ }^{2}$ Department of Pathology, Duke University Medical Center, Durham, NC, USA

Correspondence: Dr H Levinson, MD, Departments of Pathology and Surgery, Duke University Medical Center, 3181, Durham, NC 27710, USA.

E-mail: Howard.levinson@duke.edu

Received 26 April 2010; revised 14 August 2010; accepted 3 September 2010
} 
mechanisms stimulate fibroblasts and myofibroblasts to exert tractional strain. The ability of a cell to contract it's matrix is determined by the presence of a contractile apparatus that consist of bundles of specialized adhesion complexes, non-muscle myosin and actin microfilaments. ${ }^{8}$ Fibroblasts contain two different isoforms of actin, $\beta$-cytoplasmic actin and $\gamma$-cytoplasmic actin, whereas myofibroblasts also express $\alpha$-smooth muscle actin $(\alpha-$ sma, the actin isoform typically seen in vascular smooth muscle cells). ${ }^{9}$

Fibroblasts and myofibroblasts sense the physical properties of their three-dimensional environment-indirect stresses from nearby fibroblasts pulling on the woven lattice work of collagen fibers and direct stresses from cell-cell contactsand respond to the changes in elasticity by translating mechanical forces and deformations into biochemical signals. These signals adjust cytoskeletal structure and activate actomyosin contractility. The myofibroblast is widely believed to be the cell responsible for wound and scar contracture. ${ }^{8,10}$ Non-muscle myosin II (NMMII) is a protein in both fibroblasts and myofibroblasts that has an important role in several cellular processes that require force and translocation, such as migration, adhesion and contractility. ${ }^{11-14}$ NMMII activation is the end target of every known contractile signaling cascade as it is regulated by phosphorylation and activation of myosin regulatory light chain. In mammals there are at least three different NMMII heavy chains, encoded by three different genes, termed NMM IIA, IB and IIC. The isoforms are well conserved with an $80-89 \%$ homologous identity in amino acids between NMMIIA and IIB. ${ }^{15,16}$ Despite considerable homology, these isoforms seem to have unique, specialized non-redundant functions, exhibiting differences in enzymatic properties, subcellular localization and tissue expression patterns. ${ }^{17-19}$ IIA has a lower duty ratio IIB in in vitro kinetic studies, and as such, IIA likely has a role in rapid contractility, whereas IIB probably primarily serves to maintain isometric tension. ${ }^{17,20-23}$ IIA is involved in Rho-dependent function, actin stress fiber formation and focal adhesion formation (known pro-contractile factors), but IIB is not. ${ }^{19,23,24}$ IIA null cells are poor at contracting fibrin gels compared with IIB small interfering RNA knockdowns. ${ }^{13}$ Appreciating and delineating the differences between each isoform is important for identifying the isoform that is primarily responsible for mediating fibrocontractile disease and, thus, is a target for preventing disease progression.

The hypothesis is that, the change in the elasticity of the extracellular matrix (ECM) regulates IIA and IIB during the remodeling phase of cutaneous repair to promote scar contracture formation. This study demonstrates that IIA and IIB protein expression is temporally upregulated during the remodeling phase of repair and returns to baseline at the end of repair. IIA appears to be slightly more highly expressed than IIB during the remodeling period but both are similarly distributed in scar fibroblasts. All three actin isoforms were consistently expressed throughout the remodeling phase of repair, but $\beta$ - and $\gamma$-actin were found to be widely expressed throughout the dermis and $\alpha$-smooth muscle actin was only locally expressed. Increased IIA and IIB expression in scar fibroblasts correlated with increased collagen matrix contraction in comparison to lesser IIA and IIB expression in normal dermal fibroblasts from unwounded skin. Expression of IIA protein is regulated by the elasticity of the ECM.

\section{MATERIALS AND METHODS Human Tissue}

Formalin-embedded human tissues for immunohistochemical staining were obtained from the Duke University Medical Center Department of Pathology repository of tissue specimens in accordance with DUMC IRB protocols. Selection of specimen was based on the disease process-scars excision of various ages. A sample section of the tissue was stained by hematoxylin and eosin, and reviewed a under light microscope for the presence of the scar and normal tissues within the section, by a dermatopathologist. Specimens without adequate area of disease and normal tissue were excluded from selection as the normal area provides an excellent internal control to compare against (better than matched for age, gender, location and other factors). Sample size needed for each group was calculated using preliminary data, to achieve the sample needed to have $80 \%$ power to detect a difference in each group's respective means, using a paired Student's $t$-test with a 0.05 two-sided significant level.

\section{Immunohistochemistry (IHC)}

Consecutive sections of $5 \mu \mathrm{m}$ for each tissue specimen were mounted on silanized charged slides and allowed to dry for $30 \mathrm{~min}$ in an incubator at $60^{\circ} \mathrm{C}$. After deparaffinization in xylene and rehydration, slides were covered for $10 \mathrm{~min}$ with $3 \%$ hydrogen peroxide to block endogenous peroxidase. Slides were then placed in citrate antigen-retrieval buffer for $20 \mathrm{~min}$ at $80^{\circ} \mathrm{C}$, followed by washing in tris-buffered saline tween-20 (0.5 M Tris Base, $9 \% \mathrm{NaCl}, 0.5 \%$ Tween 20 and $\mathrm{pH}$ 8.4 ) at room temperature. Slides were then placed in a humid chamber and incubated for $45 \mathrm{~min}$ with the primary antibodies (rabbit anti-myosin IIA, rabbit anti-myosin IIB, mouse anti- $\alpha$-sma, $\beta$-actin and $\gamma$-actin, at 1:100 dilution Abcam. Cambridge, MA, USA). After three rinses in trisbuffered saline tween-20, the slides were incubated for $45 \mathrm{~min}$ with biotinylated secondary detection antibodies (goat antirabbit or anti-mouse at 1:200 dilution, Vector Laboratories, Burlingame, CA, USA). After three rinses in tris-buffered saline tween-20 the slides were incubated with the detection system (Vectastain Elite ABC, Vector Laboratories) for $30 \mathrm{~min}$. Tissue staining was visualized with a DAB substrate chromogen solution (Innovex, Richmond, CA, USA). Slides were counterstained with hematoxylin, dehydrated and mounted. Negative controls were performed by using a rabbit polyclonal IgG (for IIA and IIB) or mouse polyclonal IgG (for actin isoforms) at matched concentration. 


\section{Cell Culture}

Fibroblasts from explants of human skin tissues were obtained as whole-tissue surgical specimen from the operating room per approved IRB protocol. Specimens for fibroblast isolation were all taken from scar excision procedures, the age of scars are given in Table 3. Scar with surrounding normal tissues was easily distinguished, divided and explanted separately using previously described protocol. ${ }^{25}$ In brief, tissues were washed, finely minced and incubated in collagenase type I with $1 \%$ penicillin/streptomycin (SigmaAldrich, St Louis, MO, USA) at $37^{\circ} \mathrm{C}$ for $24-48 \mathrm{~h}$. The cells were subsequently cultured in DMEM supplemented with $10 \%$ FBS and 1\% penicillin/streptomycin (Sigma). Experiments with primary cell cultures were performed when cells have become $80-90 \%$ confluent between passage 1 and 6 .

\section{Fibroblast Populated Collagen Lattice (FPCL)}

The fabrications of both free-floating FPCLs (FF-FPCL) and stressed FPCLs (S-FPCL) were followed as described previously. ${ }^{26}$ For FF-FPLC, $400 \mu \mathrm{l}$ of fibroblast-collagenmedium mixture at concentration of $1.0 \times 10^{5} \mathrm{cells} / \mathrm{ml}$, $1.28 \mathrm{mg} / \mathrm{ml}$ purified collagen, (Nutragen, Advanced Biomatrix, Fremont, CA, USA) was added into a 24-well flatbottom tissue culture plate that had been blocked with BSA. The plate was placed in a $37^{\circ} \mathrm{C}$ incubator with $5 \% \mathrm{CO}_{2}$ for $60 \mathrm{~min}$ to allow the collagen to polymerize, before adding $500 \mu$ l of conditional solution $0.5 \%$ BSA in DMEM, 10\% FBS in DMEM and $10 \mu \mathrm{M}$ blebbistatin (Calbiochem, La Jolla, CA, USA) in 0.5\% BSA/DMEM, which freed lattices from the surface. The sizes of lattices were recorded using a digital scanner $4 \mathrm{~h}$ after release, and the areas of each lattice were determined and measured using Image $(\mathrm{NIH}$, Bethesda, MD, USA). All conditions were performed in triplicate per experiment, and these experiments were repeated three times. For S-FPCL, $400 \mu \mathrm{l}$ volumes of a fibroblast-collagen-medium mixture at a concentration of $1.0 \times 10^{5}$ cells per $\mathrm{ml}$ and increasing collagen concentration-0.64, 1.28, 2.56 and $4.83 \mathrm{mg} / \mathrm{ml}$ were added into a 24-well flat-bottom tissue culture plate. The collagen was allowed to polymerize, as described above, before adding $1 \mathrm{ml}$ of growth media $(10 \%$ FBS in DMEM). On day 4, cells were harvested from the lattice for western assay. All conditions were performed in triplicates per experiment, and these experiments were repeated three times.

\section{Western Blotting}

Detection of IIA and IIB in S-FPCL were measured by western analysis. After 4 days, cells were freed from lattice using collagenase type I ( 100 unit in DMEM incubated at $37^{\circ} \mathrm{C}$ for $30 \mathrm{~min}$ ). Cell extracts were prepared with SDS sample buffer and boiled for $10 \mathrm{~min}$ at $90^{\circ} \mathrm{C} .5 \mu \mathrm{g}$ of cell lysates were separated on $4-12 \%$ Bis-Tris gel (Invitrogen, Carlsbad, CA, USA). After electrophoresis, the separated proteins were transferred to a nitrocellulose membrane (Invitrogen). The membrane was incubated with blocking buffer for $1 \mathrm{~h}$ and then incubated with the primary antibody at $4^{\circ} \mathrm{C}$ overnight. The blots were subsequently incubated with the appropriate secondary antibody in blocking buffer. Proteins were visualized using Odyssey Infrared Imager (Model 9120, Li-Cor Inc., Lincoln, NE, USA).

\section{Polyacrylamide Substrata}

A number of methods have been developed for the preparation of elastic substrata with different stiffnesses to study the interactions between the cell and the substratum. In this study polyacrylamide substratum was used as described by Pelham and Wang (1997). ${ }^{27}$ Briefly, thin sheets of polyacrylamide were cast between a glass slide and a coverslip. To achieve a substratum of $1 \mathrm{kPa}, 5 \%$ acrylamide and $0.1 \%$ $\mathrm{N}, \mathrm{N}$-methylene bisacrylamide (BIS) were mixed with distilled water and HEPES (final concentration $10 \mathrm{mM}$ ). Polymerization is induced by adding $1 / 200$ volume of $10 \%$ ammonium persulfate (Bio-Rad) and 1/2000 volume of TEMED ( $N, N, N, N^{\prime}$-tetramethylethylenediamine, Bio-Rad) to the degassed solution, to ensure reliable polymerization. Sulfosuccinimidyl-6-(4'-azido-2'-nitrophenylamino) hexanoate (Pierce Chemical, Rockford, IL, USA) was used to cross-link collagen to the surface of the polyacrylamide substratum. The polyacrylamide substratum was then soaked in growth media for $1 \mathrm{~h}$ before inoculation with cells. Normal dermal fibroblasts $\left(2 \times 10^{5}\right)$ from three different patients were added to the polyacrylamide gel and cultured for $48 \mathrm{~h}$ before IIA and IIB analysis with flow cytometry.

\section{Flow Cytometry}

To analyze cells for IIA or IIB by flow cytometry single-cell suspensions were prepared by trypsinization, and were fixed and permeabilized using Cytofix/Cytoperm fixation/permeabilization kit (BD Biosciences, San Diego, CA, USA). Cells were stained with IIA and IIB antibodies (at 1:200 dilution, Santa Cruz Biotechnology, Santa Cruz, CA, USA), followed by fluorescein isothiocyanate-conjugated anti-rabbit IgG (Jackson ImmunoResearch, West Grove, PA, USA) in $\mathrm{Ca}^{2+}$ - and $\mathrm{Mg}^{2+}$-free BSA/HBSS. Samples were analyzed on a Guava EasyCyte Plus flow cytometer (Guava Technologies, Hayward, CA, USA). A total of 10000 cells were analyzed in each sample, and data were collected using CytoSoft Data Acquisition and Analysis Software (Guava Technologies). Data were analyzed and the geometric mean of the fluorescence intensity for each cell type was calculated using Flowjo version 7.6 (Tree Star Inc., Ashland, OR, USA).

\section{Statistical Analysis}

All values are presented as mean \pm s.e.m. Statistical analysis was performed using JMP software (SAS Institute ver. 7, Cary, NC, USA). Differences between groups were compared by Student's $t$-test and are considered to be statistically significant at values of $P \leq 0.05$. Mean inhibition of NMMII from blebbistatin on normal and scar samples were tested using ANOVA, with $P$-value $\leq 0.05$ is statistically significant. 


\section{RESULTS}

\section{Spatiotemporal Expression of NMMII in Human Scar During the Remodeling Phase of Repair}

A total of 33 human scar samples with surrounding normal skin spanning the remodeling phase of repair, ranging in scar age from 14 to 1058 days were selected for IHC staining. Samples were selected to match for patient's race, gender, age and scar location. Specimens were equivalent across groups $(P \geq 0.05)$ except for gender. There were 32 samples scar samples obtained from caucasian patients and 1 sample from a black patient, because of the high prevalence of specimens obtained for melanoma (Tables 1 and 2).

Table 1 Patient demographic of selected scar samples

\begin{tabular}{lrr}
\hline Group & $N$ & $P$-value \\
\hline Race & & \\
Caucasian & 32 & $<0.05$ \\
Black & 1 & \\
Gender & & NS \\
Male & 17 & \\
Female & 16 & \\
Age (years) & & NS \\
$<50$ & 18 & \\
$>50$ & 15 & NS \\
Scar location & & \\
Head & & \\
Trunk & 6 & \\
Upper extremities & 12 & \\
Lower extremities & 8 & \\
\hline
\end{tabular}

NS, not significant.

${ }^{\mathrm{a}}$ Significantly more scar samples were obtained from white patient because of the higher prevalence of conditions, such as melanoma that needed re-excision for confirmation or recurrences.

Table 2 Scar ages at the time of excision

\begin{tabular}{lcc}
\hline Age & $N$ & $P$-value \\
\hline a weeks-6 months & $\mathbf{2 4}$ & \\
Male & 12 & NS \\
Female & 12 & \\
& & \\
6 months & 9 & NS \\
Male & 5 & \\
Female & 4 &
\end{tabular}

\footnotetext{
${ }^{\mathrm{a}} P$-value of both IIA and IIB IHC staining intensity groups comparing normal and scar human tissue.
}

Figure 1 illustrates selected specimens from different time periods of scar maturation, stained by hematoxylin and eosin and IHC staining for IIA, IIB and the actin isoforms $\alpha, \beta$ and $\gamma$ (Figure 1). Note, there is an initial rise in IIA and IIB expression at the initiation of the remodeling phase ( 2 weeks), followed by the maintenance of high expression levels throughout the remodeling phase (6 weeks and 18 weeks) and lastly a resolution of IIA and IIB expression levels after the throughout remodeling phase, equivalent to normal unwounded skin (27 weeks). IIA and IIB were ubiquitously expressed throughout the scar dermis and even in the epidermis. Protein expression in all scar specimens had increased in IIA and IIB, as compared with normal surrounding skin. IIA stained more intensely than IIB in all scar samples. Human scar samples were immunostained for $\alpha$-sma, $\beta$-actin and $\gamma$-actin (Figure 1). Expression of $\alpha$-sma, $\beta$-actin and $\gamma$-actin were consistent throughout the remodeling phase of repair, following a similar trend to NMMII. However, although $\beta$-actin and $\gamma$-actin were highly expressed throughout the dermis, $\alpha$-sma was only focally expressed in select dermal fibroblasts.

High-power magnification $(100 \times)$ of IIA and IIB staining revealed that both isoforms were expressed throughout the cytoplasm of activated fibroblasts (Figure 2). There were no differences in subcellular localization patterns of either isoform in activated fibroblasts. The nuclei in scar fibroblasts appeared plump and active compared with the quiescent appearing, comma-shaped nuclei of unwounded skin, IIA and IIB were localized to a peri-nuclear location in wounded skin or quiescent fibroblasts.

\section{NMMIIA and NMMIIB Expression in Scar Fibroblasts Correlates with Increased Contractility}

Patient-matched scar and normal human dermal fibroblasts were explanted from seven surgical specimens and analyzed for IIA and IIB protein levels using flow cytometry (Table 3). Figure 3 depicts IIA and IIB flow cytometry results from a single scar and normal fibroblast cell line from the patient sample. The data show a consistently maintained increase in both IIA and IIB expression levels in in vitro maintained scar fibroblasts as compared with normal fibroblasts (Figure 3).

The in vitro cell-based FPCL is a frequently used model to study three-dimensional tissue remodeling, in which cell behavior in S-FPCL or FF-FPCL contraction closely mimics what is observed in vivo. ${ }^{28-32}$ The seven paired cell lines (normal and scar) were placed into FF-FPCL and cultured in the presence of basal media with $0.5 \%$ BSA for $6 \mathrm{~h}$ (Figure 4 ). In all seven samples, scar fibroblasts produced more collagen matrix contraction than matched dermal fibroblasts $(P \leq 0.05)$. These data suggested that IIA and IIB expression correlates with increased tissue remodeling.

The same seven matched cell pairs were placed into FFFPCL and cultured with 10\% FBS in DMEM media, and $10 \mu \mathrm{M}$ blebbistatin to determine how specific inhibition of NMMII affects scar and normal fibroblast tissue remodeling 


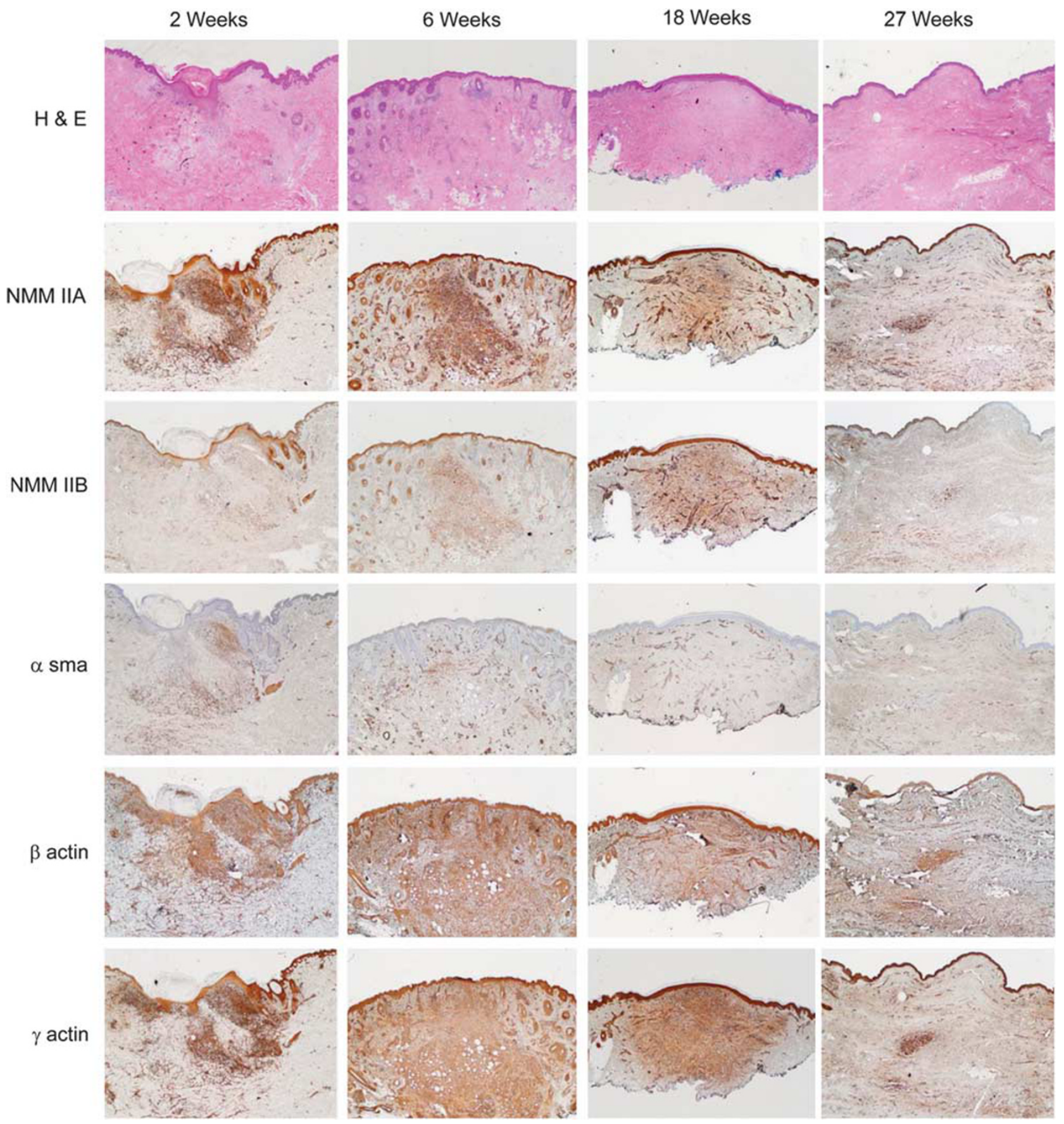

Figure 1 Non-muscle myosin isoforms IIA and IIB, and $\alpha$-smooth muscle-, $\beta$ - and $\gamma$-actin expression in the remodeling phase of repair (a). There is a robust protein expression of non-muscle myosin isoforms IIA and IIB during the remodeling period (6 to 18 weeks) compared with early (2 weeks) and late scars ( 27 weeks) at low-power views $(2 \times$ objective). $\alpha$-smooth muscle actin expression is focally expressed in the dermis throughout the remodeling phase of repair. There is a robust, widespread protein expression of $\beta$ - and $\gamma$-actin throughout the remodeling phase of repair. Negative controls are rabbit and mouse non-specific immunogobulin for non-muscle myosin and actin isoforms, respectively. Scale bar $=1 \mathrm{~mm}$.

in a cytokine-rich environment. ${ }^{33}$ Scar fibroblasts populated latticed, contracted more than normal fibroblasts with statistical significance being reached in three primary cells. Blebbistatin significantly inhibited collagen matrix contraction by $84.9 \pm 1.9 \%$ in normal fibroblasts versus $77.5 \pm 1.8 \%$ in scar fibroblasts, demonstrating that NMMII was causative of contractility $(P \leq 0.05)$.

\section{Increased Mechanical Tension-Induced Non-Muscle Myosin II Expression}

NMMII is upregulated in scar tissue throughout the remodeling phase of repair with a resolution to baseline levels at the end of remodeling (Figures 1-3); yet, it is unclear what are the mechanisms that may be contributing to this biological phenotype. One possible causative factor is 

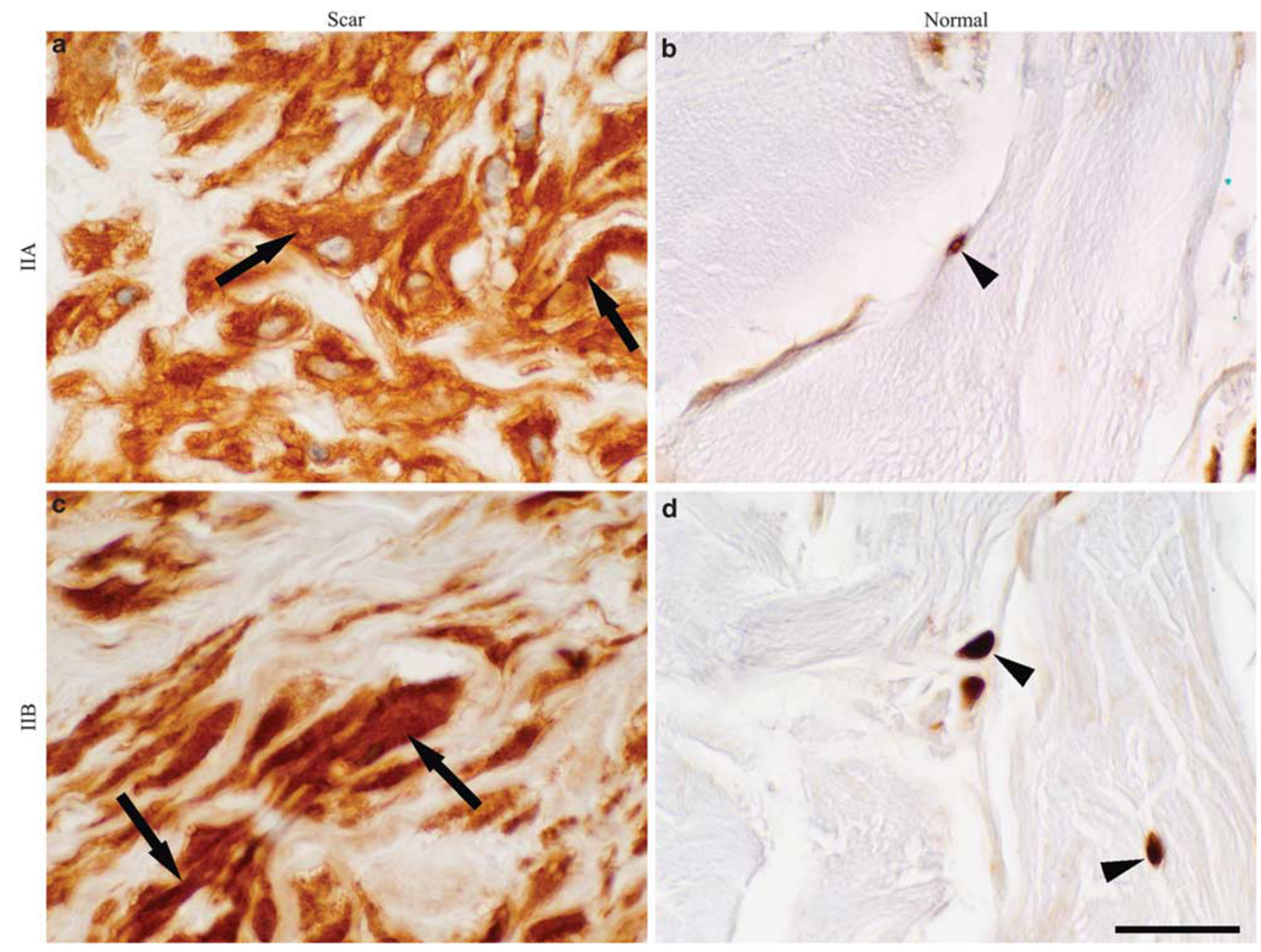

Figure 2 Representative histological sections of non-muscle myosin isoforms IIA and IIB in scar and matched normal human skin. High-power objectives $(100 \times)$ of human scar samples with normal skin tissue stained for IIA $(\mathbf{a}, \mathbf{b})$ and IIB $(\mathbf{c}, \mathbf{d})$. There is a significant difference in IIA and IIB protein localization within mesenchymal cells in scar compared with normal skin tissue. Scar mesenchymal cells are oval with rounded nuclei and normal skin cells are spindle shaped with coma-shaped nuclei. Non-muscle myosin is more robust and widely distributed in the cytoplasm in scar fibroblasts (black arrows) compared with peri-nuclear localization in normal skin cells (arrowheads). Scale bar $=10 \mu \mathrm{m}$.

mechanotransduction. To investigate how changes in extracellular mechanical tension alter IIA and IIB expression, fibroblasts were cultivated in S-FPLC for 4 days. Fibroblasts were embedded in stressed collagen lattices consisting of a range of different collagen concentrations $0.64,1.28,2.56$ and $4.83 \mathrm{mg} / \mathrm{ml}$ for 4 days. Western analysis shows that there is a consistent increase in IIA protein levels with increasing collagen concentration; however, IIB protein level does not significantly change (Figure 5). Lattices consisting of different collagen concentrations have been shown to correlate with increasing fibroblast stress. ${ }^{34}$ However, the physical properties of the lattices are undefined and variable. In addition to collagen lattices, we used polyacrylamide gels where the concentration of bis-acrylamide determines the elasticity. ${ }^{27,35}$ We determined the elasticity of the polyacrylamide gels in this study to be $\sim 1 \mathrm{kPa}$ using atomic force microscopy (data not shown). Fibroblasts were cultured on polyacrylamide gels expressed less IIA than fibroblasts cultured on glass microscope slides coated with collagen (Figure 5b). IIB was not found to increase significantly $(P \leq 0.05)$.

\section{DISCUSSION}

Scar contracture is an incremental, progressive shortening process that occurs over months to years after injury, dominated by extracellular events, such as matrix remodeling, ${ }^{8}$ resulting in tissue deformities, physiological impairment and displeasing esthetics.

It is well accepted that the contractile activity of fibroblasts and myofibroblasts is primarily responsible for connective tissue remodeling during wound healing; however, the contribution of each cell to contracture formation and the functional difference between the two cell phenotypes remains unclear. Nevertheless, these issues become less important as the mechanisms of cell contractility and tissue remodeling of fibroblasts and myofibroblasts share the common motor protein NMMII. Our IHC data of human 
Table 3 Scar ages at the time of fibroblast explantation

\begin{tabular}{lc}
\hline Age (weeks) & Sample \\
\hline 84 & A \\
62 & B \\
6 & C \\
10 & D \\
18 & E \\
36 & F \\
84 & G
\end{tabular}

Fibroblasts were explanted from the scar (ages as indicated) and surrounding normal tissue as detailed in Materials and Methods.
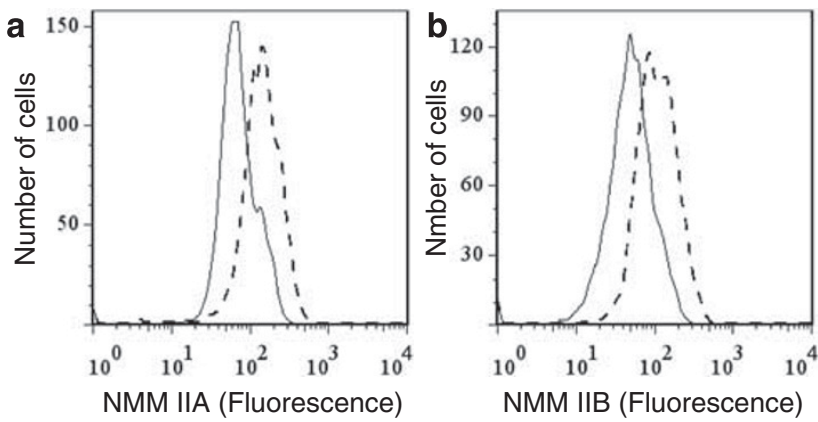

C

\begin{tabular}{c|c|c}
\multirow{2}{*}{ Patient Sample } & \multicolumn{2}{c}{$\begin{array}{c}\text { Mean Fluorescence Intensity Increase } \\
\text { (Scar-Normal) }\end{array}$} \\
\cline { 2 - 3 } & NMM IA & NMM IIB \\
\cline { 2 - 3 } A & $31.26 \pm 2.56$ & $39.99 \pm 8.06$ \\
B & $8.56 \pm 1.12$ & $61.38 \pm 19.2$ \\
C* & $\mathbf{5 7 . 0 0 \pm 9 . 7 0}$ & $\mathbf{4 8 . 8 6} \pm \mathbf{2 . 9 0}$ \\
D & $66.33 \pm 4.12$ & $78.86 \pm 26.35$ \\
E & $234.26 \pm 2.43$ & $158.81 \pm 20.11$ \\
F & $62.19 \pm 5.66$ & $24.72 \pm 2.19$ \\
G & $12.46 \pm 2.87$ & $12.76 \pm 7.06$
\end{tabular}

Figure 3 Non-muscle myosin IIA and IIB protein level in explanted human scar and normal skin. Flow cytometry data show a representative increase in IIA (a) and IIB (b) protein level in an explanted normal and scar fibroblasts from the same patient. This increase is seen in all seven scar and normal pairs of human explanted fibroblasts (c). ${ }^{*}$ denotes patient sample with representative histograms shown.

scar tissues shows significant increase in protein level of IIA and IIB during the remodeling phase of wound healing that declines after 6 months. This rise and fall in protein expression through the remodeling period are observed across patients' gender, age and scar location, signifying NMMII importance in tissue remodeling and perhaps as a conserved physiological response to wound repair and scar contracture. Furthermore, this differential expression pattern reveals that in addition to being regulated by myosin regulatory light chain, NMMII is also regulated at the transcription and translation levels.
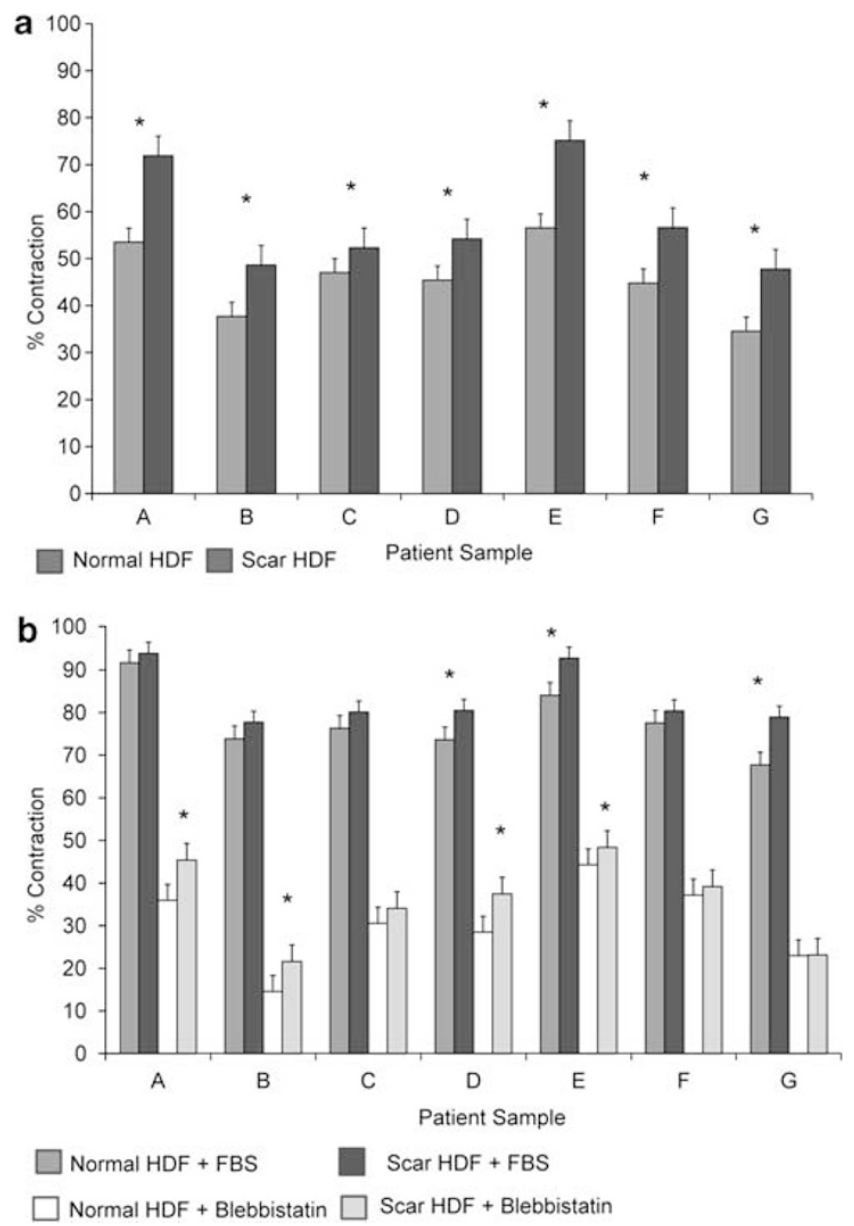

Figure 4 Scar fibroblasts contract collagen matrices faster than normal skin fibroblasts in free-floating (FF)-FPCL. Explanted fibroblasts were placed into FF-collagen lattices with lattice area measured after $6 \mathrm{~h}$. Under basal condition (0.5\% BSA in DMEM), scar fibroblasts display significantly greater contraction than normal fibroblasts in all seven sample pairs (a). Under stimulated condition (10\% FBS in DMEM), means percent lattice contraction are greater in scars in all samples, but only three pairs with statistical significant difference in means (b). Inhibition of NMMII using $10 \mu \mathrm{M}$ blebbistatin decreases overall contraction in all samples, but scar fibroblasts continue to have greater contraction than normal fibroblasts $(\mathbf{b}) .{ }^{*}$ denotes statistical significance $(P$-value $\leq 0.05)$.

The myofibroblast has widely become regarded as the cell responsible for scar contracture. However, this is controversial as it has been shown that human sacroccygeal pilonidal sinus wounds contract without a high density of myofibroblasts being present and in vitro studies reveal that fibroblasts exhibit contractile activity. ${ }^{36,37}$ Data presented in this paper indicates that $\beta$ - and $\gamma$ - actin are highly expressed throughout the dermis and they have a similar temporal profile during 2-27 weeks of scar maturation to that of IIA and IIB, whereas $\alpha$-sma is only locally expressed in dermal fibroblasts during this same time period. These data indicate that the interactions of IIA and IIB with $\beta$ - and $\gamma$-actin may account for tissue remodeling and that $\alpha$-sma may not be necessary for contraction as previously thought. 
a

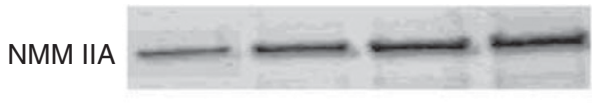

NMM IIB

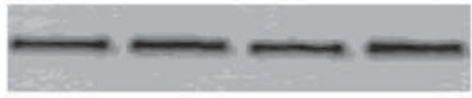

GAPDH

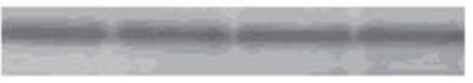

ECM

Tension
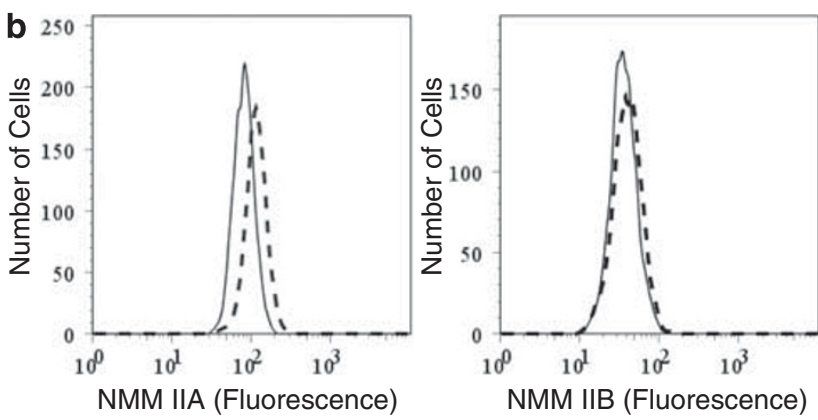

Figure 5 Extracellular matrix mechanical tension upregulates non-muscle myosin isoform IIA. Three different normal human dermal fibroblast cell lines were seeded in stressed collagen type I matrix at increasing mechanical tension using increasing collagen concentration-0.64, 1.28, 2.56 and $4.83 \mathrm{mg} / \mathrm{ml}$. Lattices were left anchored for 4 days to maximize isometric tension development. Western data show that mechanical strain causes an increase in IIA protein expression, but not IIB (a). Representative western blots IIA and IIB, and GAPDH are shown. Normal dermal fibroblasts cultured on glass slides or polyacrylamide gels with stiffness of $1 \mathrm{kPa}$, for $48 \mathrm{~h}$. Fibroblasts were stained for IIA and IIB and analyzed by flow cytometry. Flow cytometric data shows that IIA expression was increased when fibroblasts were cultured on glass (dashed lines) compared with polyacrylamide gels (solid lines, b).

The increase of NMMII also correlates to an increase in in vitro cell contractility as evidenced by our FF- and S-FPCL data. As mentioned, cell behavior in FPCL contraction closely mimics what is observed in vivo as focal adhesions formation, ECM remodeling and isotonic contraction observed in FPCL contraction parallels the findings in contracting wounds and scars. $^{29-31}$ Acellular collagen lattices do not contract. Our data show that scar fibroblasts, with a higher level of NMMII, are able to contract collagen matrix more than normal fibroblasts with lower NMMII protein level under minimal and stimulated condition in both FF- and S-FPCL. Fibroblasts from other fibrotic diseases, such as pulmonary fibrosis and Crohn's disease, have been shown to have increased contractility by a comparative amount of $15-25 \%$ in collagen lattices compared with normal fibroblasts. ${ }^{38,39}$ To show that NMMII is indeed the motor protein responsible for cell contractility, lattice contraction was observed in the presence of blebbistatin. Blebbistatin has been shown to be a potent inhibitor of NMMII, but not some muscle myosin II isoforms or unconventional myosins class $\mathrm{I}, \mathrm{V}$ and $\mathrm{X} .{ }^{40}$ It functions through binding to the large cleft of the motor domain and interfering with the opening and closing of the myosin during the contractile state. ${ }^{40,41}$ As blebbistatin inhibits cell contractility by nearly $80 \%$ in both FF- and S-FPCL, it is likely that the contractile forces are generated by NMMII.

In addition to the molecular composition of cell contractility, the mechanical property of the ECM has an important influence on the morphology and function of fibroblast, including synthetic, proteolytic and contractile activities, and the secretion of ECM proteins in response to injury. ${ }^{42-46}$ In most normal connective tissues, fibroblasts are protected from external tensile stress by their surrounding ECM. ${ }^{47}$ The meshwork of collagen fibers, elastic fibers and glycosaminoglycans in dermis can be subjected to large deformations, which are not directly transmitted to the embedded resident fibroblasts owing to the lack of extensive cell-matrix contacts. Hence, in normal conditions, fibroblasts are stress-shielded and do not develop extensive contractile protein as our IHC data have shown. This situation changes dramatically when the ECM is altered, such as after dermal injury, when the wound is provisionally filled with inflammatory cells that release different cytokines that stimulates fibroblasts to form contacts with the ECM and migrate into the wound bed. ${ }^{48}$ These fibroblasts can be resident fibroblasts or derived from precursors of another origin, such as epithelial or endothelial cells, circulating fibrocytes and bone-marrow derived precursors. ${ }^{8,49-54}$ The origins and the pathways involved in the differentiation into scar fibroblasts with increased NMM expression are unknown.

Fibroblasts proliferate and start to restore the dermis by secreting and organizing the characteristic dermal ECM; however, the increasing number of fibroblasts enhances matrix rigidity and further reinforcement of cell-matrix contacts. In addition, the disrupted ECM, together with the increase in cell-matrix contacts have effectively removed any stress-shielding fibroblasts had from high extracellular tensile strain from the newly deposited, immature, un-cross-linked and non-remodeled collagen. ${ }^{55}$ In response to increased environmental strain, fibroblasts upregulate its contractile apparatus (for example, focal adhesions and NMMII) to reorganize and contract its surrounding matrix toward restoring homeostatic balance. This response is consistent with our IHC data showing the level of NMMII significantly increases at the beginning of the remodeling process of repair.

In general, cells sense their physical environment by translating mechanical forces and deformations-by inputs from ECM, neighboring cells and physical stress-into biochemical signals that can adjust cellular and extracellular structure and functions. This mechanosensitive feedback modulates cellular functions, as diverse as proliferation, differentiation, migration and apoptosis, as well as being crucial for organ development and homeostasis. Any molecular defect that interrupts or alters this chain of mechanical sensing and subsequent cell-signaling events could perturb 
the normal cellular function and potentially lead to diverse diseases, such as loss of hearing, cardiovascular disease, muscular dystrophy and cancer. To study the effect of mechanical tension on fibroblasts' contractile protein NMMII, human dermal fibroblasts were embedded in collagen lattices of different collagen concentration or cultured on polyacrylamide gels with a stiffness of $1 \mathrm{kPa}$. Willits et al have shown in their studies the stiffness of the collagen gels significantly increase with increasing collagen concentration. ${ }^{34}$ Using these experimental models, our data show an increase in IIA expression with increasing mechanical tension; however, the level of IIB remains relatively constant. The differential response of IIA and IIB to mechanical tension may be due to the unique functions each NMMII isoform possesses. Studies on the biochemical functions of each NMMII isoform support the notion that IIA is likely the main protein for promoting scar contracture: NMMIIA has a lower duty ratio than NMMIIB in in vitro kinetic studies, and as such, IIA likely has a function in rapid contractility, whereas IIB probably primarily serves to maintain isometric tension. ${ }^{17,20,22,23}$ In addition, IIA is involved in known procontractile factors, such as Rho-dependent function, actin stress fiber formation and focal adhesion formation, but IIB is not. ${ }^{19,23,24}$ IIA-null cells are poor at contracting fibrin gels compared with IIB small interfering RNA knockdowns. ${ }^{13}$ Despite these speculative differences in the two isoforms' functions, the mechanism behind the lack of IIB response to mechanical tension cannot be fully explained on the basis of this assay. Further study is also needed to objectively quantify the mechanical strain of the collagen lattices to better correlate it to in vivo scar environment, but nevertheless, our result does show a positive association between environmental strain and cell contractility.

As the remodeling phase progresses, the perpetual deposition, cross-linking of collagen fibers, and matrix compaction by fibroblasts create parallel arrays of collagen fibers that is better able to stress-shield the cells. ${ }^{8}$ As a result, fibroblasts are unable to sense the high extracellular strain, and thus revert back to a more quiescent state. This downregulation of contractile proteins correlates with our IHC data showing a decline in NMMII toward baseline level after 6 months.

In summary, our findings show that non-muscle myosin II is an important motor protein in cell contractility and tissue remodeling that has the potential to mediate scar contracture should a dysregulation occurred in the healing process. Furthermore, our results indicate a positive relationship between the mechanical strain of the extracellular microenvironment and non-muscle myosin II expression level, implicating it as a possible mechanism behind the partial effective of pressure therapy in post-burn scar contracture. This has also led us to believe that specific inhibition of nonmuscle myosin II during the remodeling phase of repair would effectively prevent and stop scar contracture from development.

\section{ACKNOWLEDGEMENTS}

The project was supported by a Mentored Clinical Scientist Development Award (K08), from the National Institute of General Medical Sciences, National Institutes of Health and a Plastic Surgery Education Foundation Fresh Start Surgical Gifts Fellowship. Supplemental support was provided by the Division of Plastic and Reconstructive Surgery and Departments of Pathology and Surgery at Duke University (Grant \# GM085562-01).

$\mathrm{Li} \mathrm{CaO}, \mathrm{PhD}$ assisted with atomic force microscopy measurements.

\section{Disclaimer}

The contents of this manuscript are solely the responsibility of the authors and do not necessarily represent the official views of the $\mathrm{NIH}$.

\section{DISCLOSURE/CONFLICT OF INTEREST}

The authors declare no conflict of interest.

1. Renovo. Renovo Ltd.: Biotechanalytics, 2001; http://www.renovo.com/ content.asp?c_id=16, accessed 13th Oct 2010.

2. Nedelec B, Ghahary A, Scott PG, et al. Control of wound contraction. Basic and clinical features. Hand Clin 2000;16:289-302.

3. Stadelmann WK, Digenis AG, Tobin GR. Physiology and healing dynamics of chronic cutaneous wounds. Am J Surg 1998;176(2A Suppl):26S-38S.

4. Clark RA. Cutaneous tissue repair: basic biologic considerations. I. J Am Acad Dermatol 1985;13(5 Part 1):701-725.

5. Hanley WS, Snyder ME, Field L, et al. Dose-response studies of penicillamine and related compounds in reducing skin-tensile strength of rats. Chem Biol Interact 1978;21:263-270.

6. Levinson H, Moyer KE, Saggers GC, et al. Calmodulin-myosin light chain kinase inhibition changes fibroblast-populated collagen lattice contraction, cell migration, focal adhesion formation, and wound contraction. Wound Repair Regen 2004;12:505-511.

7. Lawrence WT. Physiology of the acute wound. Clin Plast Surg 1998;25:321-340.

8. Tomasek JJ, Gabbiani G, Hinz B, et al. Myofibroblasts and mechanoregulation of connective tissue remodelling. Nat Rev Mol Cell Biol 2002;3:349-363.

9. Gabbiani G. The biology of the myofibroblast. Kidney Int 1992;41: 530-532.

10. Majno G, Gabbiani G, Hirschel BJ, et al. Contraction of granulation tissue in vitro: similarity to smooth muscle. Science 1971;173: 548-550.

11. Abe $\mathrm{M}$, Ho $\mathrm{CH}$, Kamm KE, et al. Different molecular motors mediate platelet-derived growth factor and lysophosphatidic acid-stimulated floating collagen matrix contraction. J Biol Chem 2003;278: 47707-47712.

12. Meshel AS, Wei $\mathrm{Q}$, Adelstein RS, et al. Basic mechanism of threedimensional collagen fibre transport by fibroblasts. Nat Cell Biol 2005;7:157-164.

13. Even-Ram S, Doyle AD, Conti MA, et al. Myosin IIA regulates cell motility and actomyosin-microtubule crosstalk. Nat Cell Biol 2007;9:299-309.

14. Vicente-Manzanares M, Ma X, Adelstein RS, et al. Non-muscle myosin II takes centre stage in cell adhesion and migration. Nat Rev Mol Cell Biol 2009;10:778-790.

15. Golomb E, Ma X, Jana SS, et al. Identification and characterization of nonmuscle myosin II-C, a new member of the myosin II family. J Biol Chem 2004;279:2800-2808.

16. Simons $M$, Wang $M$, McBride OW, et al. Human nonmuscle myosin heavy chains are encoded by two genes located on different chromosomes. Circ Res 1991;69:530-539.

17. Kovacs $M$, Wang $F, \mathrm{Hu} A$, et al. Functional divergence of human cytoplasmic myosin II: kinetic characterization of the non-muscle IIA isoform. J Biol Chem 2003;278:38132-38140.

18. Tullio AN, Accili D, Ferrans VJ, et al. Nonmuscle myosin II-B is required for normal development of the mouse heart. Proc Natl Acad Sci USA 1997;94:12407-12412.

19. Conti MA, Even-Ram S, Liu C, et al. Defects in cell adhesion and the visceral endoderm following ablation of nonmuscle myosin heavy chain II-A in mice. J Biol Chem 2004;279:41263-41266. 
20. Wylie SR, Chantler PD. Separate but linked functions of conventional myosins modulate adhesion and neurite outgrowth. Nat Cell Biol 2001;3:88-92.

21. Wylie SR, Wu PJ, Patel H, et al. A conventional myosin motor drives neurite outgrowth. Proc Natl Acad Sci USA 1998;95:12967-12972.

22. Saitoh T, Takemura S, Ueda K, et al. Differential localization of nonmuscle myosin II isoforms and phosphorylated regulatory light chains in human MRC-5 fibroblasts. FEBS Lett 2001;509:365-369.

23. Lo CM, Buxton DB, Chua GC, et al. Nonmuscle myosin Ilb is involved in the guidance of fibroblast migration. Mol Biol Cell 2004;15:982-989.

24. Sandquist JC, Swenson KI, Demali KA, et al. Rho kinase differentially regulates phosphorylation of nonmuscle myosin II isoforms $A$ and B during cell rounding and migration. J Biol Chem 2006;281: 35873-35883.

25. Takashima A. Establishment of fibroblast cultures. Curr Protoc Cell Biol 2001; 2.1.1-2.1.12

26. Paul Ehrlich H, Sun B, Kainth KS, et al. Elucidating the mechanism of wound contraction: rapid versus sustained myosin ATPase activity in attached-delayed-released compared with free-floating fibroblastpopulated collagen lattices. Wound Repair Regen 2006;14:625-632.

27. Pelham Jr RJ, Wang Y. Cell locomotion and focal adhesions are regulated by substrate flexibility. Proc Natl Acad Sci USA 1997;94:13661-13665.

28. Gross J, Farinelli W, Sadow P, et al. On the mechanism of skin wound 'contraction': a granulation tissue 'knockout' with a normal phenotype. Proc Natl Acad Sci USA 1995;92:5982-5986.

29. Carlson MA, Longaker MT. The fibroblast-populated collagen matrix as a model of wound healing: a review of the evidence. Wound Repair Regen 2004;12:134-147.

30. Bell $E$, Ivarsson B, Merrill C. Production of a tissue-like structure by contraction of collagen lattices by human fibroblasts of different proliferative potential in vitro. Proc Natl Acad Sci USA 1979;76:1274-1278.

31. Ehrlich HP. Wound closure: evidence of cooperation between fibroblasts and collagen matrix. Eye 1988;2( Part 2):149-157.

32. Carlson MA, Longaker MT, Thompson JS. Modulation of FAK, Akt, and p53 by stress release of the fibroblast-populated collagen matrix. J Surg Res 2004;120:171-177.

33. Limouze J, Straight AF, Mitchison T, et al. Specificity of blebbistatin, an inhibitor of myosin II. J Muscle Res Cell Motil 2004;25:337-341.

34. Willits RK, Skornia SL. Effect of collagen gel stiffness on neurite extension. J Biomater Sci Polym Ed 2004;15:1521-1531.

35. Pelham Jr RJ, Wang Y. High resolution detection of mechanical forces exerted by locomoting fibroblasts on the substrate. Mol Biol Cell 1999;10:935-945.

36. Berry DP, Harding KG, Stanton MR, et al. Human wound contraction: collagen organization, fibroblasts, and myofibroblasts. Plast Reconstr Surg 1998;102:124-131; discussion 132-124.

37. Wrobel LK, Fray TR, Molloy JE, et al. Contractility of single human dermal myofibroblasts and fibroblasts. Cell Motil Cytoskeleton 2002; 52:82-90.

38. Zhang HY, Gharaee-Kermani M, Zhang $\mathrm{K}$, et al. Lung fibroblast alphasmooth muscle actin expression and contractile phenotype in bleomycin-induced pulmonary fibrosis. Am J Pathol 1996;148: 527-537.

39. Regan MC, Flavin BM, Fitzpatrick JM, et al. Stricture formation in Crohn's disease: the role of intestinal fibroblasts. Ann Surg 2000;231:46-50.

40. Allingham JS, Smith R, Rayment I. The structural basis of blebbistatin inhibition and specificity for myosin II. Nat Struct Mol Biol 2005;12: 378-379.

41. Straight AF, Cheung A, Limouze J, et al. Dissecting temporal and spatial control of cytokinesis with a myosin II Inhibitor. Science 2003;299:1743-1747.

42. Kessler D, Dethlefsen S, Haase I, et al. Fibroblasts in mechanically stressed collagen lattices assume a 'synthetic' phenotype. J Biol Chem 2001;276:36575-36585.

43. Chiquet $M$. Regulation of extracellular matrix gene expression by mechanical stress [In Process Citation]. Matrix Biol 1999;18:417-426.

44. Grinnell $\mathrm{F}$, Ho $\mathrm{CH}$. Transforming growth factor beta stimulates fibroblast-collagen matrix contraction by different mechanisms in mechanically loaded and unloaded matrices. Exp Cell Res 2002;273: 248-255.

45. Hinz $B$, Mastrangelo $D$, Iselin $C E$, et al. Mechanical tension controls granulation tissue contractile activity and myofibroblast differentiation. Am J Pathol 2001;159:1009-1020.

46. Mudera VC, Pleass R, Eastwood M, et al. Molecular responses of human dermal fibroblasts to dual cues: contact guidance and mechanical load. Cell Motil Cytoskeleton 2000;45:1-9.

47. Silver FH, Siperko LM, Seehra GP. Mechanobiology of force transduction in dermal tissue. Skin Res Technol 2003;9:3-23.

48. Grotendorst GR, Grotendorst CA, Gilman T. Production of growth factors (PDGF \& TGF-beta) at the site of tissue repair. Prog Clin Biol Res 1988;266:47-54.

49. Brittan $M$, Hunt $T$, Jeffery $R$, et al. Bone marrow derivation of pericryptal myofibroblasts in the mouse and human small intestine and colon. Gut 2002;50:752-757.

50. Direkze NC, Forbes SJ, Brittan $M$, et al. Multiple organ engraftment by bone-marrow-derived myofibroblasts and fibroblasts in bone-marrowtransplanted mice. Stem Cells 2003;21:514-520.

51. Mori L, Bellini A, Stacey MA, et al. Fibrocytes contribute to the myofibroblast population in wounded skin and originate from the bone marrow. Exp Cell Res 2005;304:81-90.

52. Claudinot $S$, Nicolas $M$, Oshima $H$, et al. Long-term renewal of hai follicles from clonogenic multipotent stem cells. Proc Natl Acad Sci USA 2005;102:14677-14682.

53. Taylor G, Lehrer MS, Jensen PJ, et al. Involvement of follicular stem cells in forming not only the follicle but also the epidermis. Cell 2000;102:451-461.

54. Abe R, Donnelly SC, Peng $\mathrm{T}$, et al. Peripheral blood fibrocytes: differentiation pathway and migration to wound sites. J Immunol 2001;166:7556-7562.

55. Marenzana $M$, Wilson-Jones $\mathrm{N}$, Mudera $\mathrm{V}$, et al. The origins and regulation of tissue tension: identification of collagen tension-fixation process in vitro. Exp Cell Res 2006;312:423-433. 